\title{
When it does hurt to try: Adult age differences in the effects of instructions on implicit pattern learning
}

\author{
DARLENE V. HOWARD \\ Georgetown University, Washington, D.C. \\ and \\ JAMES H. HOWARD, JR. \\ The Catholic University of America, Washington, D.C.
}

\begin{abstract}
Trying to learn sometimes impairs implicit learning of artificialgrammars and of control systems. We asked whether such negative effects of trying also occur in implicit learning of subtle sequential regularities and whether such effects vary with adult age. Young $(n=12$, age $=20-23)$ and older $(n=24$, age $=60-80$ ) adults completed an alternating serial response time task in which predictable pattern events alternated with random ones in a visual/spatial display. Half of the participants were informed about the pattern and were instructed to try to discover it (intentional instructions), and half were not (incidental instructions). Age-related deficits in implicit learning occurred for both conditions. In addition, for the older group, but not for the younger one, intentional instructions impaired implicit pattern learning. This negative effect of trying to learn demonstrates another similarity among implicit learning tasks, supporting the view that some common processes underlie different forms of implicit learning.
\end{abstract}

Often, people set out to discover and learn about patterns, and they are subsequently able to describe them (e.g., the sequence of events required to start a car or to $\log$ on to a computer). But more often, people become sensitive to regularities without intending to do so and without being able to describe them (e.g., the syntactic regularities of language, the characteristic ways in which a friend reacts to situations). In the latter cases, implicit learning has occurred (e.g., Reber, 1993). By its nature, implicit learning usually goes unnoticed, but it is probably more important for survival than its explicit counterpart, because it enables organisms to adapt to new environments just by being in them. The present research focuses on how instructions to try to learn affect implicit acquisition and asks whether such effects vary with adult age.

Implicit learning is usually studied via three experimental paradigms that are, at least superficially, very dif-

Some of the data from the incidental condition were reported in Howard and Howard (1997), and a preliminary report of some of the present findings was included in a poster presentation at the American Psychological Society meetings in Washington, D.C., May 1997. This research was supported by Grants R37 AG02751 and R37 AG15450 from the National Institute on Aging. We thank Kim Gallagher, Sarah Oldham, and Amy Pettit for their assistance in data collection. Correspondence concerning this article should be addressed to D. V. Howard, Department of Psychology, Georgetown University, Washington, DC 20057-1001 (e-mail: howardd@ georgetown.edu)or to J. H. Howard, Jr., Department of Psychology, The Catholic University of America, Washington, DC 20064 (e-mail: howard@ cua.edu). ferent from each other: artificial grammar learning, control of complex systems, and serial response time (SRT) tasks. One counterintuitive characteristic of implicit learning has been demonstrated in the first two of these domains: Conscious attempts to discover and learn a pattern sometimes impede learning about it, at least when the pattern is nonsalient. In the earliest such demonstration, for example, Reber (1976) had college students memorize strings of letters that had been generated by a finite-state grammar. Reber (1976) found that students who had been told at the beginning that there was a regularity they should try to discover subsequently showed poorer ability to discriminate grammatical from nongrammatical strings than did students who were uninformed.

Similar findings have been demonstrated in the control of complex systems task, in which people attempt to control the output of a computer-simulated organization (e.g., the production of a sugar factory) or person (e.g., the mood of a hypothetical person). Participants who are told that there is a systematic relation between their own responses and the outputs of the system and that they should try to discover this relation sometimes are poorer at learning to control the system than are participants who are told only that they should try to control the outputs (e.g., Berry \& Broadbent, 1988).

Reber and his colleagues have noted that these negative effects of trying to learn suggest that relatively complex, nonsalient structure is best acquired in a passive manner. This conclusion runs counter to the problem- 
solving literature, where trying helps rather than hinders, and thereby supports the claim that implicit learning is distinct from explicit learning (Reber, Kasin, Lewis, \& Cantor, 1980).

One unresolved question concerns the extent to which implicit learning itself is unitary. Evidence from cognitive neuroscience suggests multiple implicit systems, with different implicit tasks calling on at least partially different neural circuits (Prull, Gabrieli, \& Bunge, 2000), but some theorists have argued for a degree of generality, assuming that all forms of implicit learning share a common process, the detection of covariation among events (e.g., Reber, 1993).

This latter common-process view suggests that the negative effects of trying should also be demonstrable in the third major implicit learning paradigm, the SRT task introduced by Nissen and Bullemer (1987). In the original version, when a target appears in one of four locations marked horizontally across a computer screen, people are to push the key under it. This terminates the target, causing the next target to appear, and so on. The target follows a repeating pattern of locations, although the participant is not informed of this fact. After several such patterned blocks, people are switched to a random sequence. An increase in response time and/or errors upon removal of the pattern demonstrates that it has been learned. Such an increase often occurs in the absence of any ability to describe the pattern, hence evidencing implicit learning.

Interestingly, the negative effects of trying to learn have not been demonstrated with this paradigm. Intentional instructions to discover and learn have usually helped when the pattern is simple and have had no effect when the pattern is more complex and subtle (e.g., Buchner, Steffens, Erdfelder, \& Rothkegel, 1997; Curran, 1997; Curran \& Keele, 1993; Frensch \& Miner, 1994; Jimenez, Mendez, \& Cleeremans, 1996). Should this be viewed as evidence against the common-process view? The SRT task does differ from the others in requiring rapid motor responses to presented stimuli. It is possible that the mechanisms underlying the negative effects of trying, which are as yet unspecified, do not apply under such conditions. For example, researchers using both artificial grammar learning and control of complex systems tasks have shown that participants in the intentional groups generate incorrect hypotheses, which then interfere with making what they think are lucky guesses but which are, in fact, based on correct, implicit knowledge (e.g., Berry \& Broadbent, 1988; Reber, 1976). It is possible, then, that this is the only mechanism underlying the negative effects of trying and that the need for rapid motor responses to presented stimuli in the SRT task prevents such interference from happening.

An alternative possibility is that the negative effects of trying are at least partially due to capacity limits; that is, trying to discover the pattern might drain mental capacity from a covariation-detecting mechanism underlying all forms of implicit learning. Detecting covariations does appear to require cognitive capacity, in that covariations among events can be learned only if these events are activated simultaneously in working memory (e.g., Cleeremans \& Jimenez, 1998; Curran, 1997; Frensch \& Miner, 1994). Consistent with this assumption, the amount of implicit learning has been shown to correlate positively with capacity measures, such as digit span or working memory span (e.g., Frensch \& Miner, 1994; Howard \& Howard, 1997). Furthermore, when the pattern is relatively complex, performing a dual task, such as detecting tones, interferes with learning, or at least the expression of learning, in the SRT task (e.g., Curran \& Keele, 1993; Frensch, Lin, \& Buchner, 1998). According to this interpretation, then, the negative effects of trying have not been seen in previous research with the SRT because the regularities to be detected were simple enough that the covariation-detecting device was not sufficiently taxed to be hurt by the simultaneous demands of trying to discover the pattern.

Aging provides a good test case for this hypothesis, because there are age-related deficits in processing capacity, as assessed, for example, by working memory span and the speed of processing (e.g., Salthouse, 1996; Zacks, Hasher, \& Li, 2000). According to the simultaneity mechanism of cognitive aging proposed by Salthouse, fewer items are simultaneously available for processing by older people than by younger ones, owing to cognitive slowing; this slower cognitive processing results in items' being activated more slowly and, thus, in decay in the activation of earlier items before later ones are fully activated. In addition, it has been shown that older people have more trouble than younger at performing two tasks at once. For example, trying to memorize a list of words while walking has minimal negative consequences for young people, but walking and memorizing interfere with each other for older people (Lindenberger, Marsiske, \& Baltes, 2000).

Given these age-related deficits in processing capacity, therefore, if trying hurts implicit learning because it drains capacity from a covariation-detecting mechanism, negative effects of trying on sequential implicit learning might appear for older people even though they usually do not for younger ones. To find out, we compared the performance of young and old adults, who were given either incidental or intentional learning instructions, on a variation of the SRT task.

In our variation (the alternating serial response time [ASRT] task), alternate targets follow a predetermined spatial pattern, whereas the intervening targets are selected randomly (Howard \& Howard, 1997). For example, a person assigned the pattern 1432 (where 1 stands for the leftmost position and 4 for the rightmost) would encounter the following series of trials, where " $r$ " stands for a randomly chosen position: $1 \mathrm{r} 4 \mathrm{r} 3 \mathrm{r} 2 \mathrm{r} 1 \mathrm{r} 4 \mathrm{r} 3 \mathrm{r} 2$, and so on. Thus, predictable pattern targets alternate with unpredictable random ones.

The ASRT has several advantages over the original (see Howard \& Howard, 1997, pp. 635-636). One is that 
the alternating random targets typically prevent participants from becoming aware of the pattern, thus enabling a relatively pure assessment of implicit learning in the absence of declarative knowledge and also ensuring that the pattern is nonsalient, a condition shown in the artificial grammar learning and control systems literature to be essential for yielding the negative effects of trying to learn. A second advantage of the ASRT is that implicit learning measures, or trial type effects (i.e., the difference in response time or in accuracy between random vs. pattern trials), are obtained throughout the experiment, rather than having to wait until a random block is presented. Hence, we can establish when a participant (or a group) first shows pattern learning by determining when the trial type effect first becomes significant, thus providing a sensitive measure of the rate of implicit learning. A third advantage of the ASRT is that, unlike the original task, in which learning could be based on lower level regularities (such as which individual items or pairs of items occur more frequently), only second-order and higher sequence structure occurs in the ASRT, since all individual events and event pairs are equally likely.

\section{METHOD}

\section{Participants}

There were 36 participants, 12 young and 24 old, with half of each group being assigned to the intentional and half to the incidental instructions. The young group consisted of undergraduate students recruited from Georgetown University, and the older consisted of community-dwelling volunteers. Their characteristics are shown in Table 1. Factorial (age $\times$ instructions) analyses of variance indicated that the instructional groups did not differ on any of these characteristics $(p>.10)$. However, as is typical, the younger groups had higher digit-symbol scores $[F(1,31)=82.62, p<.0001]$ and computational spans $[F(1,32)=23.53, p<.0001]$ than did the older, differences presumably reflecting the reduced processing ca-

Table 1

Mean Participant Characteristics, With Range

\begin{tabular}{|c|c|c|c|c|}
\hline \multirow[b]{2}{*}{ Characteristic } & \multicolumn{2}{|c|}{ Young } & \multicolumn{2}{|c|}{ Old } \\
\hline & $M$ & Range & $M$ & Range \\
\hline \multicolumn{5}{|c|}{ Incidental Learning } \\
\hline Number of males & \multicolumn{2}{|c|}{3} & \multicolumn{2}{|c|}{8} \\
\hline Number of females & \multicolumn{2}{|c|}{3} & \multicolumn{2}{|c|}{4} \\
\hline Age (years) & 21.00 & $20-23$ & 73.33 & $65-80$ \\
\hline Education (years) & 14.67 & $14-15$ & 16.42 & $12-20$ \\
\hline Computational span & 5.00 & $2-7$ & 2.50 & $1-5$ \\
\hline WAIS vocabulary & 55.83 & $44-62$ & 57.33 & $29-70$ \\
\hline WAIS digit/symbol & 73.17 & $62-85$ & 48.59 & $37.5-64$ \\
\hline Self-rated health* & 4.33 & $4-5$ & 4.45 & $3-5$ \\
\hline \multicolumn{5}{|c|}{ Intentional Learning } \\
\hline Number of males & \multicolumn{2}{|c|}{3} & \multicolumn{2}{|c|}{6} \\
\hline Number of females & \multicolumn{2}{|c|}{3} & \multicolumn{2}{|c|}{6} \\
\hline Age (years) & 20.33 & $20-21$ & 72.67 & $60-79$ \\
\hline Education (years) & 14.67 & $14-15$ & 15.83 & $13-19$ \\
\hline Computational span & 5.33 & $3-7$ & 2.83 & $0-5$ \\
\hline WAIS vocabulary & 50.00 & $35-60$ & 59.00 & $40-70$ \\
\hline WAIS digit/symbol & 82.67 & $78-90$ & 47.75 & $34-66$ \\
\hline Self-rated health* & 4.33 & $4-5$ & 3.75 & $2-5$ \\
\hline
\end{tabular}

*1 (poor)-5 (excellent). pacity in terms of speed (digit-symbol) and working memory (computational span) that occurs with aging.

\section{Design}

The design was a $2 \times 2 \times 2 \times 6$ (age $\times$ instructions $\times$ trial type $\times$ session) mixed factorial, with age (young vs. old) and instructions (incidental vs. intentional) varying between subjects and trial type (pattern vs. random) and session (1 through 6) varying within subjects.

\section{Procedure}

Each participant completed six sessions of the ASRT task described in the introduction, for approximately 1,600 trials per session. On each trial, a target appeared in one of four open circles displayed in a horizontal row across a computer screen. The target consisted of the circle's filling in. The person responded by pushing a corresponding key ("z", "x", “.”, or "“") beneath the target location as rapidly as possible, using the index and middle fingers of each hand. Pushing the correct key caused that target to disappear, and after a delay of $120 \mathrm{msec}$, the next target appeared. People completed 21 blocks of 90 trials in each session, with sessions being separated by no more than 2 days. Each block began with 10 random trials, followed by 80 trials in which the pattern trials alternated with random ones. At the end of each block, the computer displayed accuracy and response time feedback. The following six patterns were used, one for each of the 6 participants in each of the groups: 1r2r3r4r, 1r2r4r3r, 1r3r2r4r, 1r3r4r2r, 1r4r2r3r, and 1r4r3r2r. (The numbers 1 to 4 correspond, respectively, to the circles from left to right on the screen.) On the random trials, any of the four positions was equally likely to appear.

Additional supplemental tasks occurred at the end of each session, as has been described in Howard and Howard (1997, Experiment $2 \mathrm{~A}$ ).

At the beginning of the first session, everyone was told that our goal was "to learn more about how practice affects motor performance" and "to find out just how much people are able to speed their responses when they are given extended practice on a simple reaction time task." People in the intentional condition were told, in addition, that there would be some regularity to the sequence, in that every other target would follow a regular repeating sequence. They were told that knowing this regularity might help them to respond more quickly and that, at the end of each block, they would be asked to report to the experimenter their current best guess as to exactly what the repeating pattern was. They were told, however, that their main task was to try to improve their response times as much as possible.

\section{RESULTS}

We used a multivariate analysis of variance approach for within-subjects variables so as to avoid possible violations of sphericity. Therefore, the $F$ values reported are transformations based on Pillai's trace statistic, and the degrees of freedom for the error term are adjusted.

Figure 1 depicts the mean of median response times on correct trials, and Figure 2 depicts the mean proportion correct on pattern and random trials over sessions, separately for the incidental and the intentional groups, and for young and older people. As is typical, overall response times are longer for the older groups $[F(1,32)=$ $51.60, p<.0001]$. For all groups, overall response times get faster over sessions $[F(5,28)=42.15, p<.0001]$. Accuracy also declines over sessions $[F(5,28)=14.56, p<$ $.0001]$, although it is clear that this decline occurs pri- 


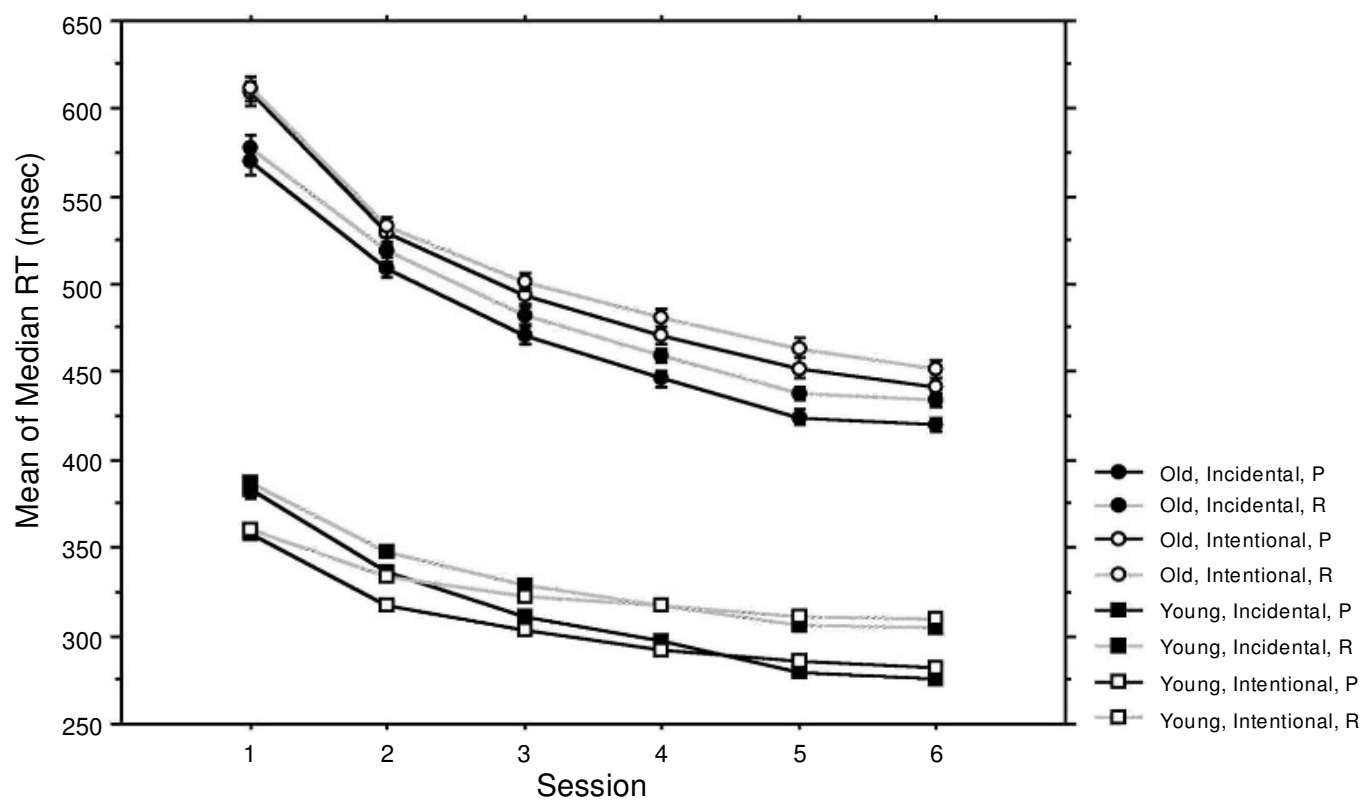

Figure 1. Mean of median correct reaction times for correct responses as a function of testing session, age, instructional condition, and trial type. Error bars of one standard error (within subjects, computed on block-level data) are plotted for each point but are only visible when greater in magnitude than the size of the symbol. In the legend, "P" stands for pattern trials, and "R" stands for random trials.

marily for random trials, rather than for pattern trials $[F(5,28)=33.32, p<.0001]$. Participants often complain that their "fingers are taking over" as training proceeds, causing them to make more errors.
The main data of interest are trial type effects (the difference between pattern and random trials), because these measure pattern learning. Such effects are difficult to discern in Figures 1 and 2, so Figure 3 depicts mean

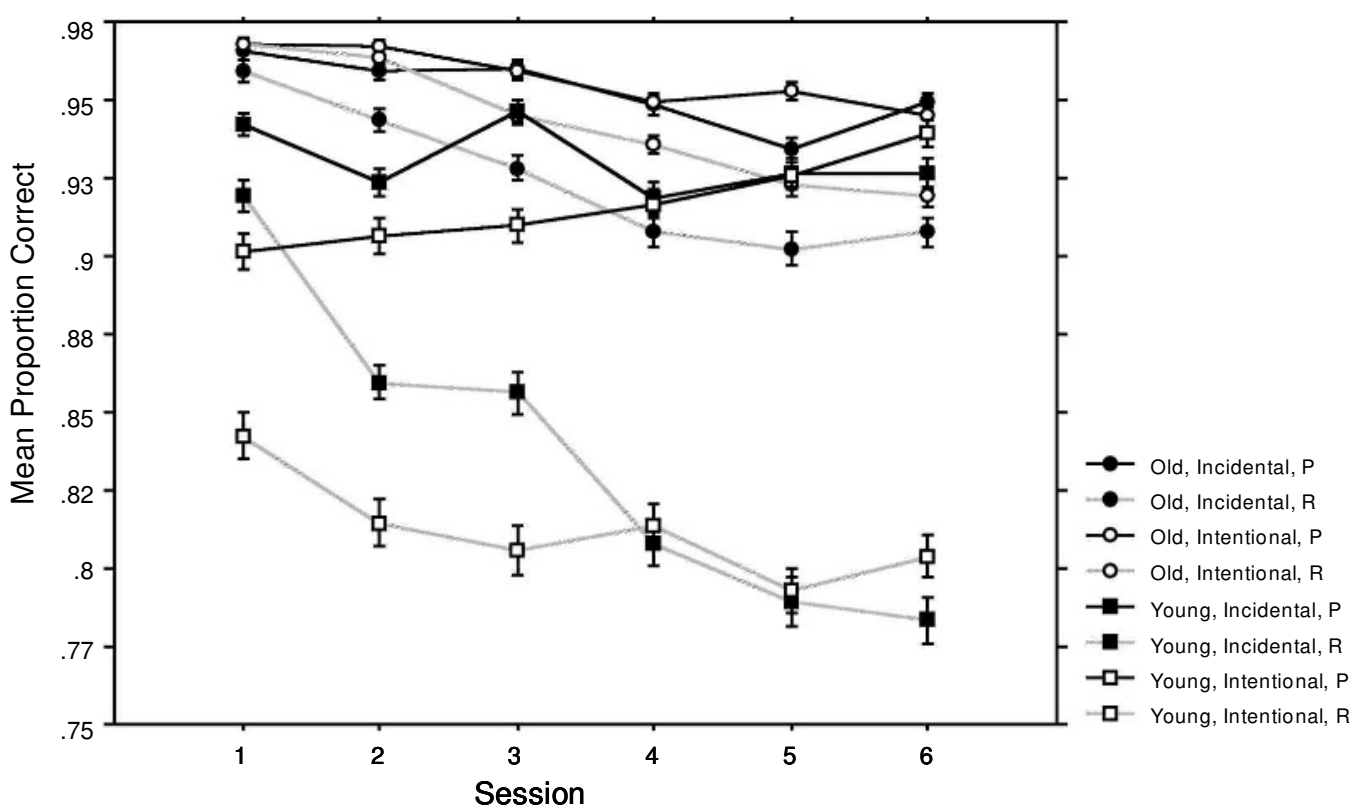

Figure 2. Mean proportion correct as a function of testing session, age, instructional condition, and trial ty pe. Error bars of one standard error (within subjects, computed on block-level data) are plotted for each point but are only visible when greater in magnitude than the size of the symbol. In the legend, "P" stands for pattern trials, and " $R$ " stands for random trials. 


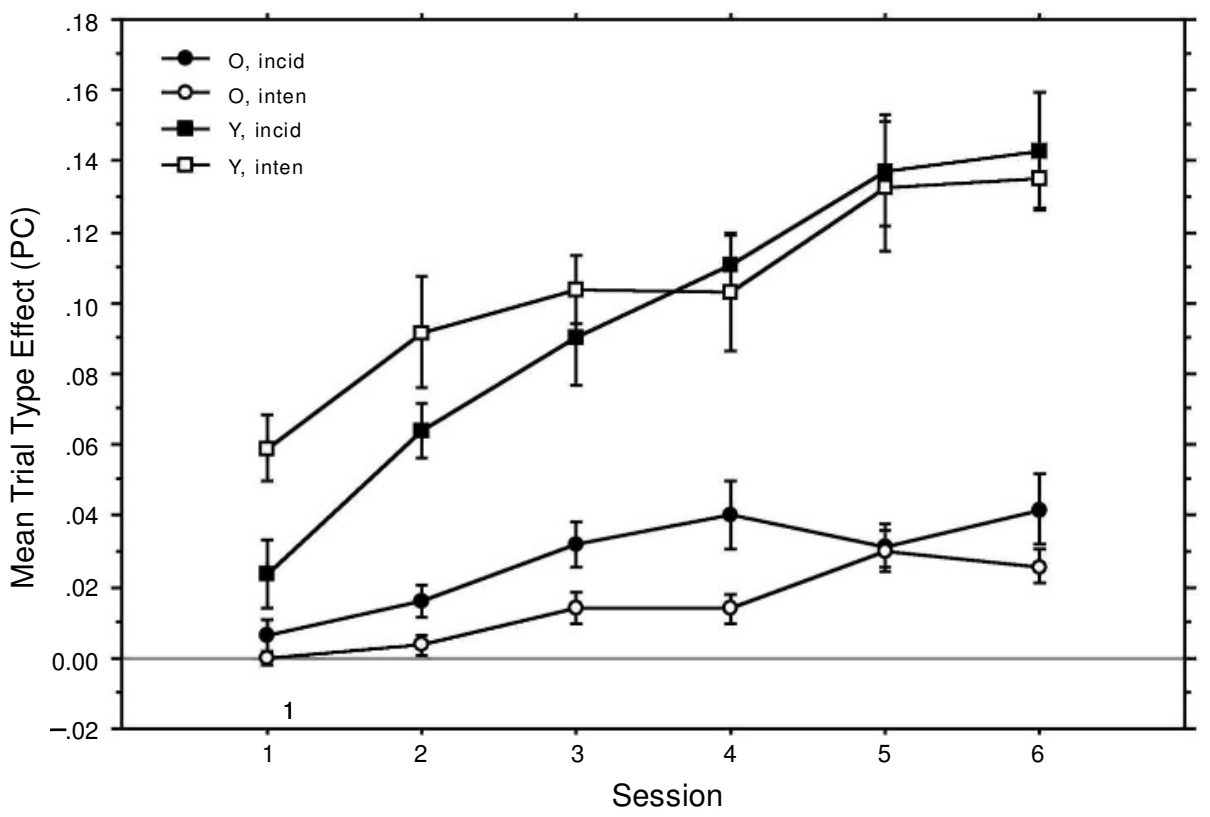

Figure 3. Mean proportion correct trial type effect (i.e., proportion correct on pattern trials minus proportion correct on random trials) for the two age groups across sessions. Data from the incidental groups are in filled symbols, and those for intentional groups are in open symbols. Error bars of one standard error are shown. O, old; Y, young.

trial type effects for the accuracy measure (i.e., proportion correct on pattern minus that on random trials) across the six sessions for each age group and instructional condition. An increase in these functions over sessions provides an indication of learning, and the difference between the incidental and the intentional function within each age group provides an indication of the effect of trying.

All analyses described here were carried out on both response time and accuracy, but because both measures yielded the same pattern of results and the accuracy measure was the more sensitive, only the accuracy trial type effects are reported.

\section{Learning on the Implicit Measures in Both Age Groups?}

As Figure 3 shows, the trial type effect increased across sessions for both age groups, signaling pattern learning. The relevant trial type $\times$ session interaction was significant for both the young $[F(5,6)=18.57, p<$ $.001]$ and the old $[F(5,18)=10.62, p<.0001]$ groups, as was the trial type effect $[F(1,10)=174.55, p<.001$, and $F(1,22)=41.61, p<.0001$, for the young and the old, respectively].

Thus, throughout the age range studied here, people are sensitive to subtle sequential regularities in their environment, consistent with previous research using the SRT and ASRT (e.g., Curran, 1997; Howard \& Howard, 1997).

\section{Age Differences on the Implicit Measures of Learning?}

Nonetheless, there are age-related deficits in pattern learning, which are apparent in Figure 3. Trial type effects increase less over sessions for the older groups than for the young ones, as is revealed by a significant age group $\times$ session $\times$ trial type interaction $[F(5,28)=10.35$, $p<.0001]$. Again, this age deficit is consistent with earlier research using relatively complex patterns (e.g., Curran, 1997; Howard \& Howard, 1997). It is also consistent with Salthouse's (1996) simultaneity mechanism: Older adults can activate fewer events simultaneously than can younger ones, thus making it more difficult for them to detect the second order regularities present in the current patterns.

\section{Age Differences in the Effects of Learning Instructions on the Implicit Measures of Pattern Learning?}

Figure 3 suggests that instructions to find the pattern influenced the age groups differently, especially early in training. Most important, and consistent with our hypothesis, the old adults show negative effects of trying, in that the intentional group shows a smaller trial type effect than does the incidental group. For the old participants, both the trial type $\times$ instructional condition interaction $[F(1,22)=4.26, p<.051]$ and the trial type $\times$ session $\times$ instructional condition interaction $[F(5,18)=$ $3.51, p<.03]$ are significant. In contrast, the young 


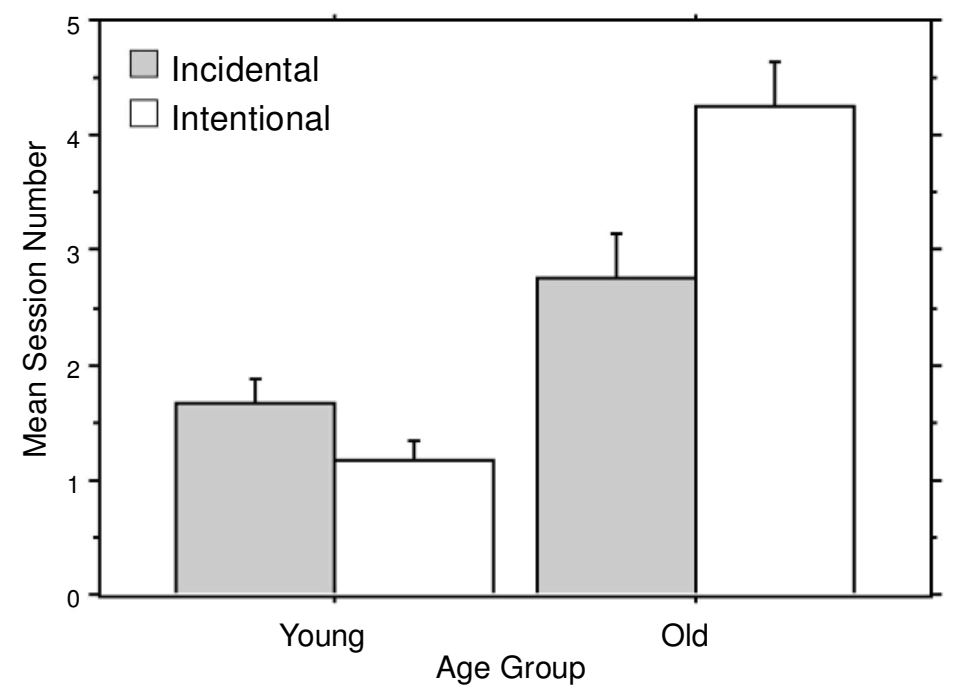

Figure 4. Mean session number on which participants of each age group and instructional condition first showed a significant trial type effect (i.e., a significant difference between pattern and random trials) on at least one of the dependent measures (i.e., either accuracy or response times). Error bars of one standard error are shown.

group reveals no significant interactions between trial type and instructional condition $(p>.10)$, and Figure 3 suggests that, at least early in training, the young intentional group is in the direction of showing a greater trial type effect than the incidental one.

These age-related effects of instructions can be seen even more clearly in Figure 4, which shows the mean session on which people in each group first showed a significant $(p<.05)$ trial type effect on either the accuracy or the response time measure - that is, the first session on which each person showed significant pattern learning. ${ }^{1}$ This interaction of age and instructional condition was statistically significant $[F(2,30)=4.24, p<$ .03]. Post hoc analyses indicated that for the older group, learning occurred on a later session under the intentional than under the incidental instructions $[t(22)=2.714, p<$ .02] but that the instructional conditions did not differ significantly for the younger people $[t(10)=1.86, p<$ $.09]$. In fact, the young people showed a trend opposite that of the older people, in that young people were in the direction of showing learning on an earlier session for intentional than for incidental instructions.

\section{Do People Gain Declarative Knowledge of the Pattern?}

In the incidental learning condition, when people were questioned at the end of their training and told that there was in fact a pattern, no one of any age was able to provide an accurate description of any part of the pattern. Thus, no one in the incidental learning condition revealed any declarative knowledge of the pattern, despite the fact that all of them had shown significant trial type effects on at least one of the measures. This suggests that in the incidental condition, the learning observed was largely implicit for both age groups.

In the intentional conditions, at the end of each block, people had been urged to give their best guess as to what their pattern was. We classified people as having full declarative knowledge if, at some point, they guessed the complete pattern correctly (i.e., they guessed $1 \mathrm{r} 2 \mathrm{r} 3 \mathrm{r} 4 \mathrm{r}$, $2 r 3 r 4 r 1 r, 3 r 4 r 1 r 2 r$ or $4 r 1 r 2 r 3 r$ if their pattern was $1 \mathrm{r} 2 \mathrm{r} 3 \mathrm{r} 4 \mathrm{r}$ ) and then did so consistently throughout the rest of the ASRT task. We classified people as having partial declarative knowledge if, at some point, they guessed at least one (but fewer than all four) of the transitions correctly (e.g., 1r2) and then kept reporting that transition consistently for the rest of training. If people never guessed even one of the correct transitions consistently, they were classified as having no declarative knowledge.

By these definitions, 2 of the 6 intentional young people gained full declarative knowledge, both at approximately the beginning of Session 5, and 2 others showed partial knowledge, in the form of getting one (and, in both cases, only one) of the transitions correct consistently, on Session 6. The remaining 2 young adults revealed no declarative knowledge. Thus, two thirds of the young people in the intentional condition were able to reveal partial or full declarative knowledge of the regularity by the end of training. In contrast, none of the 12 older people in the intentional groups was able to do so.

\section{DISCUSSION}

Our results join those from other implicit learning tasks in showing that sometimes, contrary to common 
sense, it does hurt to try. In the present case, we show that trying to learn can hurt if you are older and attempt to discover a subtle spatial/sequential regularity in the events to which you are responding.

Intentional instructions did not hurt young adults. In fact, trying to learn helped young people, in that two thirds of the intentional group eventually revealed some declarative knowledge of the regularity, and early in training the intentional group was in the direction of showing sensitivity to the pattern earlier than did the incidental one. These findings are consistent with those of earlier studies using instructional variations in the SRT task with younger participants (e.g., Buchner et al., 1997; Curran, 1997; Curran \& Keele, 1993; Frensch \& Miner, 1994; Jimenez et al., 1996). In contrast, we found that for older adults, not only did the intentional instructions fail to enable them to gain declarative knowledge of the pattern, but worse yet, these instructions slowed down the rate at which their reaction times and accuracy revealed sensitivity to the pattern.

These results demonstrate that a phenomenonnamely, the negative effects of trying to learn-which has previously been shown for grammar learning and control of complex systems, can also occur for sequential pattern learning. This is consistent with the notion that a common process, such as the detection of covariation, underlies these otherwise diverse kinds of implicit learning. According to the current hypothesis, this detection of covariation occurs even in the absence of intention to learn or of awareness of learning, but it does make demands on a limited capacity processor, in that covariation of events can be learned only if the events are activated simultaneously in memory (e.g., Cleeremans \& Jimenez, 1998; Curran, 1997; Frensch \& Miner, 1994).

Thus, we propose that one reason that trying to learn can hurt diverse forms of implicit learning is that it reduces the number of pattern events that can be activated simultaneously. According to this interpretation, our young participants did not show negative effects of trying because the simultaneous demands of trying to find the pattern and of covariation detection did not exceed their processing capacity. In contrast, these demands did exceed the more limited capacity of our older participants, thereby resulting in negative effects of trying to learn. In keeping with this explanation and with the typical findings in the cognitive aging literature, our older participants had shorter computational spans (a common measure of working memory capacity) and lower digitsymbol scores (a measure of the speed of processing) than our younger ones.

Our interpretation makes testable predictions. For example, if the pattern in an SRT task were made yet more complex (e.g., no regularities simpler than third order), thus requiring that more pattern events be activated simultaneously for learning to occur, negative effects of trying should also appear for younger people. Another prediction concerns the grammar-learning and complex systems tasks. If a common capacity-limited, covariation- detecting process underlies implicit learning in all three of these tasks, the negative effects of trying to learn that have been demonstrated for young people in grammar learning and in complex systems should be even more pronounced for older people. That is, the age $X$ instructions interaction we have observed in the ASRT task should also appear for these paradigms. We are not aware of any published experiments that test for such an interaction.

Thus, the present results are consistent with the notion that negative effects of trying on implicit learning are due, at least in part, to the increased burdens that trying places on a limited capacity covariation-detecting process. Nonetheless, other interpretations cannot be ruled out. For example, as was mentioned in the introduction, it has been shown that in the grammar-learning and complex systems tasks, young people in the intentional conditions sometimes generate and persist in using incorrect hypotheses that interfere with their making responses that are based on correct, implicit knowledge. Perhaps, then, it is this use of incorrect hypotheses that is reducing the amount of implicit learning we can detect in our older people in the intentional group, rather than their implicit learning's actually being impaired by the demands on their processing capacity. However, it seems unlikely that reliance on such incorrect hypotheses would explain the negative effects of trying that we are seeing in our older participants, because the older people were much less willing than the young to describe their hypothesis when pressed to do so at the end of each block. In fact, older people often reported that they had no hypothesis at all. Thus, we think that the incorrect hypotheses explanation for the negative effects of trying to learn cannot account for the age-selective effects we have seen here.

More generally, however, it is possible that the intentional instructions in the present study, and in earlier studies of learning artificial grammars and complex systems, hurt performance, but not implicit learning. Some SRT studies of transfer in young people suggest that the effects of dual tasks described in the introduction primarily diminish performance, not learning itself (e.g., Frensch et al., 1998). Future research will need to distinguish between these possibilities, but either of them indicates that trying to learn can sometimes compromise adaptive behavior.

In summary, we found that for older adults, but not for younger ones, attempting to discover and learn subtle spatial/sequential regularities reduced the extent to which they revealed sensitivity to those regularities. This demonstrates a similarity among diverse forms of implicit learning. The fact that such negative effects of trying appear for older, but not for younger, participants in the present study suggests that they are due to capacity limitations.

Practically speaking, adults of all ages often encounter situations that call on implicit forms of learning; they need to learn the syntax of a second language or to re- 
learn perceptual/motor skills after strokes or accidents. The negative effects of trying demonstrated here, and in studies of other forms of implicit learning, suggest that the most effective educational and rehabilitation techniques in such circumstances might be those that call on relatively passive practice and repetition, rather than on conscious awareness and hypothesis-testing strategies, especially for older adults.

\section{REFERENCES}

Berry, D. C., \& Broadbent, D. E. (1988). Interactive tasks and the implicit-explicit distinction. British Journal of Psychology, 79, 251 272.

Buchner, A., Steffens, M. C., Erdfelder, E., \& Rothkegel, R. (1997). A multinomial model to assess fluency and recollection in a sequence learning task. Quarterly Journal of Experimental Psychology, 50A, 631-663.

Cleeremans, A., \& Jimenez, L. (1998). Implicit sequence learning: The truth is in the details. In M. A. Stadler \& P. A. Frensch (Eds.), Handbook of implicit learning (pp. 323-364). Thousand Oaks, CA: Sage.

Curran, T. (1997). Effects of aging on implicit sequence learning: Accounting for sequence structure and explicit knowledge. Psychological Research, 60, 24-41.

Curran, T., \& Keele, S. W. (1993). Attentional and nonattentional forms of sequence learning. Journal of Experimental Psychology: Learning, Memory, \& Cognition, 19, 189-202.

Frensch, P. A., Lin, J., \& Buchner, A. (1998). Learning versus behavioral expression of the learned: The effects of a secondary tonecounting task on implicit learning in the serial reaction task. Psychological Research, 61, 83-98.

Frensch, P. A., \& Miner, C. S. (1994). Effects of presentation rate and individual differences in short-term memory capacity on an indirect measure of serial learning. Memory \& Cognition, 22, 95-110.

Howard, J. H., JR., \& Howard, D. V. (1997). Age differences in implicit learning of higher-order dependencies in serial patterns. Psychology \& Aging, 12, 634-656.

Jimenez, L., Mendez, C., \& Cleeremans, A. (1996). Comparing di- rect and indirect measures of sequence learning. Journal of Experimental Psychology: Learning, Memory, \& Cognition, 22, 948-969.

Lindenberger, U., Marsiske, M., \& Baltes, P. B. (2000). Memorizing while walking: Increase in dual-task costs from young adulthood to old age. Psychology \& Aging, 15, 417-436.

Nissen, M. J., \& Bullemer, P. (1987). Attentional requirements of learning: Evidence from performance measures. Cognitive Psychology, 19, 1-32.

Prull, M. W., Gabrieli, J. D. E., \& Bunge, S. A. (2000). Age-related changes in memory: A cognitive neuroscience perspective. In F. I. M. Craik \& T. A. Salthouse (Eds.), The handbook of aging and cognition (2nd ed., pp. 91-154). Mahwah, NJ: Erlbaum.

REBER, A. S. (1976). Implicit learning of synthetic languages: The role of instructional set. Journal of Experimental Psychology: Human Learning \& Memory, 2, 88-94.

Reber, A. S. (1993). Implicit learning and tacit knowledge: An essay on the cognitive unconscious. New York: Oxford University Press.

Reber, A. S., Kasin, S. M., Lewis, S., \& Cantor, G. (1980). On the relationship between implicit and explicit modes in the learning of a complex rule structure. Journal of Experimental Psychology: Human Learning \& Memory, 6, 492-502.

Salthouse, T. A. (1996). The processing-speed theory of adult age differences in cognition. Psychological Review, 103, 403-428.

ZACKS, R. T., HASHER, L., \& LI, K. Z H. (2000). Human memory. In F. I. M. Craik \& T. A. Salthouse (Eds.), The handbook of aging and cognition (2nd ed., pp. 293-358). Mahwah, NJ: Erlbaum.

\section{NOTE}

1. We determined the first session on which each person showed a significant difference between pattern and random trials for either accuracy or for response time by using blocks as observations $(d f=20)$ in a matched $t$ test. For the single person who did not reach this criterion on any of the six sessions (a 76-year-old woman in the old-old intentional group), we assigned Session 7 as her day of first learning.

(Manuscript received October 12, 1999; revision accepted for publication February 27, 2001.) 